\title{
Electron-optics systems with decreased life-time of trapped electrons for terahertz gyrotrons
}

\author{
$\underline{\text { V.N.Manuilov }}^{1,2}$, A.L.Goldenberg ${ }^{1}$, M.Yu.Glyavin ${ }^{1}$, K.A.Leshcheva ${ }^{1,2}$ \\ ${ }^{1}$ Institute of Applied Physics, Russian Academy of Sciences, N. Novgorod, Russia \\ ${ }^{2}$ Lobachevsky State University of Nizhni Novgorod, N. Novgorod, Russia, e-mail: manuilov@rf.unn.ru
}

One of the most promising applications of novel gyrotrons is a high resolution spectroscopy [1]. For this goal, scientific society needs sources with rather low microwave power (typically, from $10 \mathrm{~W}$ up to 1 KW) and frequencies $0.3-1.0 \mathrm{THz}$ or even more. For the consumer, low operating voltage is preferable. Such frequency range can be obtained mainly at the second or even third cyclotron harmonic due to present cryomagnets limitations. Same time with frequency value, frequency tunability is very important for spectroscopy applications. It is a well known, that in a "short" cavity with a reduced length of homogeneous section (and, correspondently, lower Q-factor) tuning range becomes bigger. Unfortunately, decreasing of cavity length proportionally reduce the normalized length of the interaction space and, finally, push down gyrotron efficiency. Efficiency can be restored or even increase by rise of the electron pitch-factor $g$ (ratio of gyration and longitudinal velocities) from 1.1-1.3 (at present tube) to 1.7-2.0. But in such case increase a number of electrons, reflected from the magnetic mirror (fig.1), that causes the beam instabilities. Suppression of this effect is possible by decreasing of the life-time of trapped electrons [2]. An effective way to eject trapped electrons is to install on the second anode in the triode gun the longitudinal ribs (fig.2).

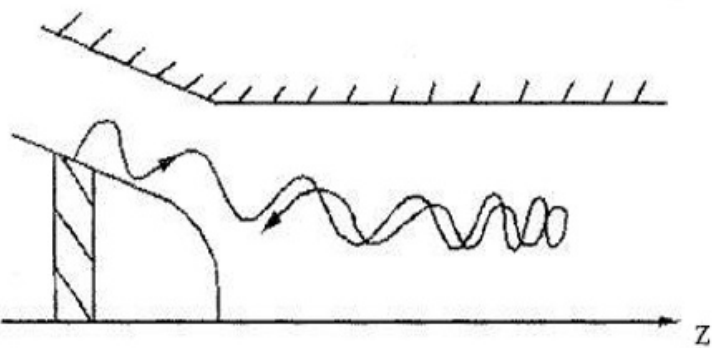

Fig. 1. The reflection of electrons from a magnetic mirror

The principle of extraction in a such gun is based on the particle drift in the crossed electric and magnetic fields. In traditional configuration of the above mentioned electron-optic system, such ribs intercept not only reflected particles, but also essential part of the primary electron beam, passed from the cathode to the cavity. To combine the small intersection of the primary beam with quick (during 1-2 longitudinal oscillations in the trap) catch of trapped electrons it is necessary to use the sectioned cathode, where emission takes place only from some sections spaced apart from azimuth angle, and/or special complex geometry of the ribs.

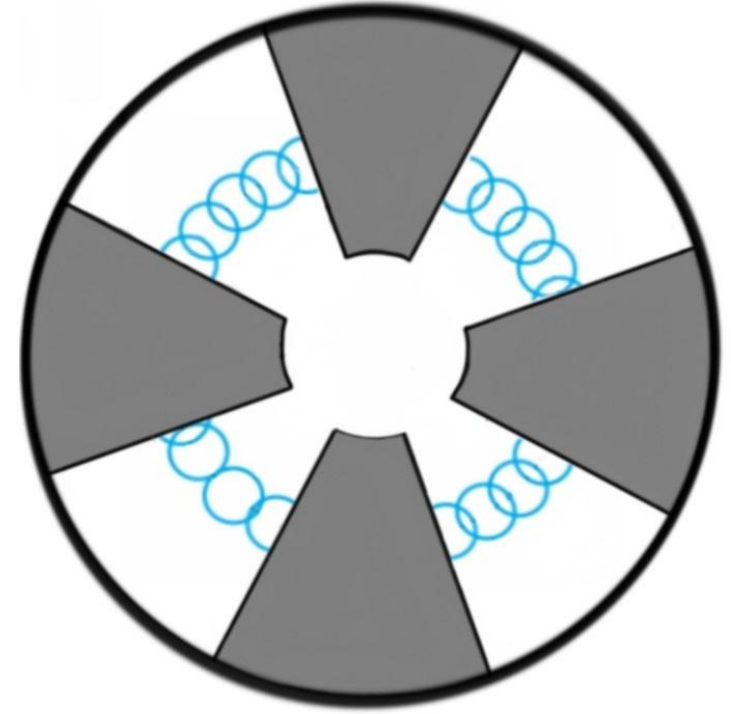

Fig. 2. The longitudinal ribs in the triode gun

In the report the preliminary geometry estimations of the sectioned electron-optic system are performed, including the profile and position of the ribs. Two versions of electron-optic system for low-voltage (16-20 kV) gyrotrons operating at the frequencies 10 $\mathrm{GHz}$ and $300 \mathrm{GHz}$ are considered. The numerical simulation of both systems is performed. The possibility to catch the trapped electrons during first 1-2 longitudinal oscillations in the trap is shown. Same time reasonable thermal load on the longitudinal ribs is obtained, good enough for CW operation. So, proposed system looks promising for the formation of the stable helical electron beam and looks reasonable for future gyrotrons for spectroscopy applications.

The reported study was funded by RFBR according to the research project № 18-32-00142

\section{References}

1. J.H.Booske, R.J.Dobbs, C.D.Joye, C.L.Kory, G.R.Neil, G.Park, J.Park, R.J.Temkin. Vacuum electronic high power terahertz sources. // IEEE Trans. on Terahertz Science and Technology, 2011, V.1, No.1. pp.54-75

2. E. V. Ilyakov, I. S. Kulagin, V. N. Manuilov, A. S. Shevchenko Experiments on the formation of an intense helical electron beam under conditions of picking-up of the electrons reflected from the magnetic mirror // Radiophysics and Quantum Electronics, 2007, Volume 50, Issue 9, pp. 713-719. 